\title{
Delay Dependent Robust Stability of Singular Systems with Additive Time-varying Delays
}

\author{
N. Chaibi \\ E. H. Tissir \\ A. Hmamed \\ Laboratory of Electronics, Signals Systems and Data Processing (LESSI), Department of Physics, \\ Faculty of Sciences Dhar El Mahraz, University Sidi Mohammed Ben Abellah, B. P. 1796, Fez-Atlas, Fez, 30000, Morocco
}

\begin{abstract}
This paper considers the problem of delay-dependent robust stability for uncertain singular systems with additive timevarying delays. The purpose of the robust stability problem is to give conditions such that the uncertain singular system is regular, impulse free, and stable for all admissible uncertainties. The results are expressed in terms of linear matrix inequalities (LMIs). Finally, two numerical examples are provided to illustrate the effectiveness of the proposed method.
\end{abstract}

Keywords: Singular systems, additive time-varying delays, linear matrix inequalities (LMIs), robust stability, delay-dependent conditions.

\section{Introduction}

Singular systems have been extensively studied in the past years due to the fact that singular systems describe physical systems better than regular ones ${ }^{[1]}$. It is also referred to as descriptor systems, implicit systems, generalized state-space systems, differential-algebraic systems or semi-state systems ${ }^{[2,3]}$.

The delay-dependent problem for singular systems is much more complicated than that for regular systems because it requires to consider not only stability, but also regularity and absence of impulses (for continuous singular systems), and causality (for discrete singular systems ${ }^{[4-7]}$ ).

In the past few years, much attention has been paid on the stability analysis of singular delay systems ${ }^{[8-18]}$. The stability and robust stabilization problem of uncertain singular time-delay systems has been studied in [19] by using the Lyapunov-Krasovskii functional and Jensen integral inequality. Gao et al. ${ }^{[14,20]}$ discussed the design of the guaranteed cost controller for the uncertain singular time-delay systems with norm-bounded parameter uncertainties. On the other hand, the delay-dependent stability and stabilization with $H_{\infty}$ prescribed performance for singular timedelay systems has been investigated ${ }^{[21,22]}$. Saadni et al. ${ }^{[1]}$ studied the robust stability and stabilization for singular systems with multiple time-varying delays by using the slack variables.

It was pointed out in [23-25] that in networked controlled system (NCS), if the signal transmitted from one point to another passes through few segments of networks, then successive delays are induced with different properties due to varying transmission conditions. Thus, it is appropriate to consider different time-delays $h_{1}(t)$ and $h_{2}(t)$ in the same state where $h_{1}(t)$ is the time-delay induced from sensor to controller, and $h_{2}(t)$ is the delay induced from controller to the actuator. The stability analysis for regular continuous systems with additive time-varying delays is studied $^{[24-26]}$. Motivated by this idea, we study the problem of robust stability for singular systems with two addi-

Manuscript received March 20, 2012; revised August 3, 2012 tive time-varying delays. We develop some delay-dependent sufficient conditions in terms of LMIs, which guarantee the singular time-delay system to be regular, impulse free, and stable. To the best of our knowledge, there is no result in the literature dealing with singular systems with additive time-varying delays.

The paper is organized as follows. In Section 2, the problem is formulated and the required lemmas are given. In Section 3, the asymptotic stability problem is established. In Section 4, we address the robust stability problem. And in Section 5, we present numerical examples to show the effectiveness of the proposed results.

\section{System description and preliminaries}

Consider an uncertain singular system described by

$$
\left\{\begin{aligned}
E \dot{x}(t)= & \left(A_{0}+\Delta A_{0}(t)\right) x(t)+ \\
& \left(A_{d}+\Delta A_{d}(t)\right) x\left(t-h_{1}(t)-h_{2}(t)\right), t>0 \\
x(t)= & \phi(t), t \in[-\bar{h}, 0]
\end{aligned}\right.
$$

where $x(t) \in \mathbf{R}^{n}$ is the state vector, $h_{1}(t)$ and $h_{2}(t)$ are the time-varying delays satisfying

$$
\begin{aligned}
& 0 \leqslant h_{1}(t) \leqslant \bar{h}_{1}, \dot{h}_{1}(t) \leqslant d_{1}, \\
& 0 \leqslant h_{2}(t) \leqslant \bar{h}_{2}, \dot{h}_{2}(t) \leqslant d_{2}, \\
& \bar{h}=\bar{h}_{1}+\bar{h}_{2}, \\
& d=d_{1}+d_{2} .
\end{aligned}
$$

The matrix $E \in \mathbf{R}^{n \times n}$ may be singular and $\operatorname{rank}(E)=r \leqslant$ $n$ is assumed. $A_{0} \in \mathbf{R}^{n \times n}$ and $A_{d} \in \mathbf{R}^{n \times n}$ are known real constant matrices, $\phi(t)$ is a compatible vector valued continuous initial function. $\Delta A_{0}(t)$ and $\Delta A_{d}(t)$ are real matrices representing parameter uncertainties, and are assumed to be norm-bounded as

$$
\left[\begin{array}{ll}
\Delta A_{0}(t) & \Delta A_{d}(t)
\end{array}\right]=D F(t)\left[\begin{array}{ll}
N_{0} & N_{d}
\end{array}\right]
$$

where $D \in \mathbf{R}^{n \times p}, N_{0} \in \mathbf{R}^{p \times n}$ and $N_{d} \in \mathbf{R}^{p \times n}$ are known real constant matrices, and $F(t)$ is a $p \times p$ unknown real 
and possibly time-varying matrix satisfying

$$
F^{\mathrm{T}}(t) F(t) \leqslant I
$$

The following lemmas are very useful for the ideas developed in this paper.

Lemma $\mathbf{1}^{[27]}$. Let $Q=Q^{\mathrm{T}}, H, E$ and $F(t)$ satisfying $F^{\mathrm{T}}(t) F(t) \leqslant I$ are appropriately dimensioned matrices, the inequality $Q+H F(t) E+E^{\mathrm{T}} F^{\mathrm{T}}(t) H^{\mathrm{T}}<0$ is true, if and only if the following inequality holds for any matrix $Y>0$, $Q+H Y^{-1} H^{\mathrm{T}}+E^{\mathrm{T}} Y E<0$.

Lemma 2 (Finsler's lemma) ${ }^{[28]}$. Consider a vector $\chi \in$ $\mathbf{R}^{n}$, a symmetric matrix $Q \in \mathbf{R}^{n \times n}$ and a matrix $B \in$ $\mathbf{R}^{m \times n}$, such that $\operatorname{rank}(B)<n$. The following statements are equivalent:

1) $\chi^{\mathrm{T}} Q \chi<0, \forall \chi$ such that $B \chi=0, \chi \neq 0$,

2) $\left.B^{\neg \mathrm{T}} Q B\right\urcorner<0$,

3) $\exists \mu \in \mathbf{R}: Q-\mu B^{\mathrm{T}} B<0$,

4) $\exists F \in \mathbf{R}^{n \times m}: Q+F B+B^{\mathrm{T}} F^{\mathrm{T}}<0$,

where $B\urcorner$ denotes a basis for the null-space of $B$.

Lemma $3^{[29]}$. For any constant matrix $M=M^{\mathrm{T}} \in$ $\mathbf{R}^{n \times n}, M>0$, scalar $\gamma \geqslant \eta(t)>0$, vector function $\omega$ : $[0, \gamma] \rightarrow \mathbf{R}^{n}$ such that the integrations in the following are well defined, then

$$
\begin{aligned}
& \eta(t) \int_{0}^{\eta(t)} \omega^{\mathrm{T}}(\beta) M \omega(\beta) \mathrm{d} \beta \geqslant \\
& {\left[\int_{0}^{\eta(t)} \omega(\beta) \mathrm{d} \beta\right]^{\mathrm{T}} M\left[\int_{0}^{\eta(t)} \omega(\beta) \mathrm{d} \beta\right] . }
\end{aligned}
$$

Consider the nominal system of (1) given by

$$
E \dot{x}(t)=A_{0} x(t)+A_{d} x\left(t-h_{1}(t)-h_{2}(t)\right) .
$$

Definition $\mathbf{1}^{[2]}$. 1) The pair $\left(E, A_{0}\right)$ is said regular if $\operatorname{det}\left(s E-A_{0}\right)$ is not identically zero. 2) The pair $\left(E, A_{0}\right)$ is said to be impulse free if $\operatorname{deg}\left(\operatorname{det}\left(s E-A_{0}\right)\right)=\operatorname{rank}(E)$.

Definition $\mathbf{2}^{[15]}$. 1) The singular time-delay system (5) is said to be regular and impulse free if the pair $\left(E, A_{0}\right)$ is regular and impulse free. 2) Singular time-delay system (5) is said to be stable if for any $\varepsilon>0$, there exists a scalar $\delta(\varepsilon)>0$, such that for any compatible initial conditions $\phi(t)$ satisfying $\sup _{-\bar{h} \leqslant t \leqslant 0}\|\phi(t)\| \leqslant \delta(\varepsilon)$, the solution $x(t)$ of system (5) satisfies $\|x(t)\| \leqslant \varepsilon$ for $t \geqslant 0$. Furthermore, $x(t) \rightarrow 0, t \rightarrow \infty$. 3) Singular time-delay system (5) is said to be admissible if it is regular, impulse-free and stable.

\section{$3 \quad$ Stability analysis}

In this section, we will present the delay-dependent stability conditions for singular systems with two additive time-varying delays. The conditions depend on the upper bound of the delays as given in (2).

Theorem 1. Given $\bar{h}_{1}>0$ and $\bar{h}_{2}>0$, then system (5) is regular, impulse free, and asymptotically stable if there exist $n \times n$ matrices $P, F_{0}, F_{1}, F_{2}$, and $n \times n$ positive definite matrices $Q_{1}, Q_{2}, Z_{1}$, and $Z_{2}$ such that the following conditions hold.

$$
\begin{gathered}
P E=E^{\mathrm{T}} P^{\mathrm{T}} \geqslant 0 \\
Q_{1}-Q_{2} \geqslant 0
\end{gathered}
$$

$$
M=\left[\begin{array}{llll}
M_{11} & M_{12} & M_{13} & M_{14} \\
* & M_{22} & M_{23} & M_{24} \\
* & * & M_{33} & M_{34} \\
* & * & * & M_{44}
\end{array}\right]<0
$$

where "*" denotes the symmetric part in a symmetric matrix, and

$$
\begin{aligned}
& M_{11}=Q_{1}-\frac{1}{\bar{h}_{1}} E^{\mathrm{T}} Z_{1} E+F_{0} A_{0}+A_{0}^{\mathrm{T}} F_{0}^{\mathrm{T}} \\
& M_{12}=\frac{1}{\bar{h}_{1}} E^{\mathrm{T}} Z_{1} E \\
& M_{13}=F_{0} A_{d}+A_{0}^{\mathrm{T}} F_{1}^{\mathrm{T}} \\
& M_{14}=P-F_{0}+A_{0}^{\mathrm{T}} F_{2}^{\mathrm{T}} \\
& M_{22}=-\left(1-d_{1}\right)\left(Q_{1}-Q_{2}\right)-\frac{1}{\bar{h}_{1}} E^{\mathrm{T}} Z_{1} E-\frac{1}{\bar{h}_{2}} E^{\mathrm{T}} Z_{2} E \\
& M_{23}=\frac{1}{\bar{h}_{2}} E^{\mathrm{T}} Z_{2} E \\
& M_{24}=0 \\
& M_{33}=-\frac{1}{\bar{h}_{2}} E^{\mathrm{T}} Z_{2} E-(1-d) Q_{2}+F_{1} A_{d}+A_{d}^{\mathrm{T}} F_{1}^{\mathrm{T}} \\
& M_{34}=-F_{1}+A_{d}^{\mathrm{T}} F_{2}^{\mathrm{T}} \\
& M_{44}=\bar{h}_{1} Z_{1}+\bar{h}_{2} Z_{2}-F_{2}-F_{2}^{\mathrm{T}} .
\end{aligned}
$$

Proof. From (8), it follows that

$$
\left[\begin{array}{ll}
M_{11} & M_{14} \\
M_{14}^{\mathrm{T}} & M_{44}
\end{array}\right]<0 .
$$

Let $J=\left[\begin{array}{ll}I & A_{0}^{\mathrm{T}}\end{array}\right]$. Pre-multiplying and post-multiplying (10) by $J$ and $J^{\mathrm{T}}$, respectively, we get

$Q_{1}-\frac{1}{\bar{h}_{1}} E^{\mathrm{T}} Z_{1} E+P A_{0}+A_{0}^{\mathrm{T}} P^{\mathrm{T}}+\bar{h}_{1} A_{0}^{\mathrm{T}} Z_{1} A_{0}+\bar{h}_{2} A_{0}^{\mathrm{T}} Z_{2} A_{0}<0$.

Now, choose two nonsingular matrices $M$ and $N$ such that

$$
\begin{aligned}
& M E N=\left[\begin{array}{ll}
I_{r} & 0 \\
0 & 0
\end{array}\right] \\
& \bar{A}_{0}=M A_{0} N=\left[\begin{array}{ll}
\bar{A}_{1} & \bar{A}_{2} \\
\bar{A}_{3} & \bar{A}_{4}
\end{array}\right] \\
& \bar{P}=N^{\mathrm{T}} P M^{-1}=\left[\begin{array}{ll}
\bar{P}_{1} & \bar{P}_{2} \\
\bar{P}_{3} & \bar{P}_{4}
\end{array}\right]
\end{aligned}
$$

and denote

$$
\begin{gathered}
\bar{Q}_{1}=N^{\mathrm{T}} Q_{1} N \\
\bar{Z}_{1}=M^{-\mathrm{T}} Z_{1} M^{-1}=\left[\begin{array}{ll}
\bar{z}_{11} & \bar{z}_{12} \\
\bar{z}_{13} & \bar{z}_{14}
\end{array}\right] \\
\bar{Z}_{2}=M^{-\mathrm{T}} Z_{2} M^{-1}=\left[\begin{array}{ll}
\bar{z}_{21} & \bar{z}_{22} \\
\bar{z}_{23} & \bar{z}_{24}
\end{array}\right] .
\end{gathered}
$$

By using (6), it can be shown that $\bar{P}_{3}=0$. Premultiplying and post-multiplying (11) by $N^{\mathrm{T}}$ and $N$, respectively, we get

$$
\begin{aligned}
& \bar{Q}_{1}-\frac{1}{\bar{h}_{1}}\left[\begin{array}{ll}
\bar{z}_{11} & 0 \\
0 & 0
\end{array}\right]+\bar{P} \bar{A}_{0}+\bar{A}_{0}^{\mathrm{T}} \bar{P}^{\mathrm{T}}+\bar{h}_{1} \bar{A}_{0}^{\mathrm{T}} \bar{Z}_{1} \bar{A}_{0}+ \\
& \bar{h}_{2} \bar{A}_{0}^{\mathrm{T}} \bar{Z}_{2} \bar{A}_{0}<0 .
\end{aligned}
$$


Since $\bar{Q}_{1}>0, \bar{h}_{1} \bar{A}_{0}^{\mathrm{T}} \bar{Z}_{1} \bar{A}_{0} \geqslant 0, \bar{h}_{2} \bar{A}_{0}^{\mathrm{T}} \bar{Z}_{2} \bar{A}_{0} \geqslant 0$, it can be easily observed that $\bar{A}_{4}^{\mathrm{T}} \bar{P}_{4}^{\mathrm{T}}+\bar{P}_{4} \bar{A}_{4}<0$, which implies that $\bar{A}_{4}$ is nonsingular, consequently, the pair $\left(E, A_{0}\right)$ is regular and impulse free. Therefore, according to Definition 1 , system (5) is regular and impulse free.

Now, we prove the stability. Let $x_{t}=x(t+\theta)$ for $\theta \in$ $[-\bar{h}, 0]$, and consider the following Lyapunov functional

$$
V\left(x_{t}\right)=V_{1}\left(x_{t}\right)+V_{2}\left(x_{t}\right)+V_{3}\left(x_{t}\right)
$$

where

$$
\begin{aligned}
V_{1}\left(x_{t}\right)= & x^{\mathrm{T}}(t) P E x(t) \\
V_{2}\left(x_{t}\right)= & \int_{-\bar{h}_{1}}^{0} \int_{t+\sigma}^{t} \dot{x}^{\mathrm{T}}(s) E^{\mathrm{T}} Z_{1} E \dot{x}(s) \mathrm{d} s \mathrm{~d} \sigma+ \\
& \int_{-\bar{h}_{1}-\bar{h}_{2}}^{-\bar{h}_{1}} \int_{t+\sigma}^{t} \dot{x}^{\mathrm{T}}(s) E^{\mathrm{T}} Z_{2} E \dot{x}(s) \mathrm{d} s \mathrm{~d} \sigma \\
V_{3}\left(x_{t}\right)= & \int_{t-h_{1}(t)}^{t} x^{\mathrm{T}}(s) Q_{1} x(s) \mathrm{d} s+ \\
& \int_{t-h_{1}(t)-h_{2}(t)}^{t-h_{1}(t)} x^{\mathrm{T}}(s) Q_{2} x(s) \mathrm{d} s .
\end{aligned}
$$

Then, the time-derivative of $V\left(x_{t}\right)$ along the solution of system (5) gives

$$
\begin{aligned}
& \dot{V}_{1}\left(x_{t}\right)=2 x^{\mathrm{T}}(t) P E \dot{x}(t) \\
& \dot{V}_{2}\left(x_{t}\right)=\int_{-\bar{h}_{1}}^{0}\left[\dot{x}^{\mathrm{T}}(t) E^{\mathrm{T}} Z_{1} E \dot{x}(t)-\right. \\
& \left.\dot{x}^{\mathrm{T}}(t+\sigma) E^{\mathrm{T}} Z_{1} E \dot{x}(t+\sigma)\right] \mathrm{d} \sigma+ \\
& \quad \int_{-\bar{h}_{1}-\bar{h}_{2}}^{-\bar{h}_{1}}\left[\dot{x}^{\mathrm{T}}(t) E^{\mathrm{T}} Z_{2} E \dot{x}(t)-\right. \\
& \left.\dot{x}^{\mathrm{T}}(t+\sigma) E^{\mathrm{T}} Z_{2} E \dot{x}(t+\sigma)\right] \mathrm{d} \sigma \\
& \dot{V}_{2}\left(x_{t}\right)=\bar{h}_{1} \dot{x}^{\mathrm{T}}(t) E^{\mathrm{T}} Z_{1} E \dot{x}(t)- \\
& \quad \int_{t-\bar{h}_{1}}^{t} \dot{x}^{\mathrm{T}}(s) E^{\mathrm{T}} Z_{1} E \dot{x}(s) \mathrm{d} s+\bar{h}_{2} \dot{x}^{\mathrm{T}}(t) E^{\mathrm{T}} Z_{2} E \dot{x}(t)- \\
& \int_{t-\bar{h}}^{t-\bar{h}_{1}} \dot{x}^{\mathrm{T}}(s) E^{\mathrm{T}} Z_{2} E \dot{x}(s) \mathrm{d} s .
\end{aligned}
$$

For any symmetric positive definite matrices $Z_{1}$ and $Z_{2}$, the following inequalities always hold ${ }^{[25]}$.

$-\int_{t-\bar{h}_{1}}^{t} \dot{x}^{\mathrm{T}}(s) E^{\mathrm{T}} Z_{1} E \dot{x}(s) \mathrm{d} s \leqslant-\int_{t-h_{1}(t)}^{t} \dot{x}^{\mathrm{T}}(s) E^{\mathrm{T}} Z_{1} E \dot{x}(s) \mathrm{d} s$

$$
-\int_{t-\bar{h}}^{t-\bar{h}_{1}} \dot{x}^{\mathrm{T}}(s) E^{\mathrm{T}} Z_{2} E \dot{x}(s) \mathrm{d} s \leqslant-\int_{t-h(t)}^{t-h_{1}(t)} \dot{x}^{\mathrm{T}}(s) \times
$$

$$
E^{\mathrm{T}} Z_{2} E \dot{x}(s) \mathrm{d} s
$$

where $h(t)=h_{1}(t)+h_{2}(t)$.

$$
\begin{aligned}
& \dot{V}_{2}\left(x_{t}\right) \leqslant \bar{h}_{1} \dot{x}^{\mathrm{T}}(t) E^{\mathrm{T}} Z_{1} E \dot{x}(t)- \\
& \int_{t-h_{1}(t)}^{t} \dot{x}^{\mathrm{T}}(s) E^{\mathrm{T}} Z_{1} E \dot{x}(s) \mathrm{d} s+\bar{h}_{2} \dot{x}^{\mathrm{T}}(t) E^{\mathrm{T}} Z_{2} E \dot{x}(t)- \\
& \int_{t-h(t)}^{t-h_{1}(t)} \dot{x}^{\mathrm{T}}(s) E^{\mathrm{T}} Z_{2} E \dot{x}(s) \mathrm{d} s
\end{aligned}
$$

By Lemma 3,

$$
\begin{aligned}
& \dot{V}_{2}\left(x_{t}\right) \leqslant \bar{h}_{1} \dot{x}^{\mathrm{T}}(t) E^{\mathrm{T}} Z_{1} E \dot{x}(t)+\bar{h}_{2} \dot{x}^{\mathrm{T}}(t) E^{\mathrm{T}} Z_{2} E \dot{x}(t)- \\
& \quad \frac{1}{\bar{h}_{1}}\left[x(t)-x\left(t-h_{1}(t)\right]^{\mathrm{T}} E^{\mathrm{T}} Z_{1} E\left[x(t)-x\left(t-h_{1}(t)\right]-\right.\right. \\
& \quad \frac{1}{\bar{h}_{2}}\left[x\left(t-h_{1}(t)\right)-x(t-h(t)]^{\mathrm{T}} E^{\mathrm{T}} Z_{2} E\left[x\left(t-h_{1}(t)\right)-\right.\right. \\
& x(t-h(t)] \\
& \dot{V}_{3}\left(x_{t}\right)=x^{\mathrm{T}}(t) Q_{1} x(t)-\left(1-\dot{h}_{1}(t)\right) x^{\mathrm{T}}\left(t-h_{1}(t)\right) Q_{1} \times \\
& x\left(t-h_{1}(t)\right)+\left(1-\dot{h}_{1}(t)\right) x^{\mathrm{T}}\left(t-h_{1}(t)\right) Q_{2} x\left(t-h_{1}(t)\right)- \\
& \left(1-\dot{h}_{1}(t)-\dot{h}_{2}(t)\right) x^{\mathrm{T}}(t-h(t)) Q_{2} x(t-h(t)) \\
& \dot{V}_{3}\left(x_{t}\right) \leqslant x^{\mathrm{T}}(t) Q_{1} x(t)-\left(1-d_{1}\right) x^{\mathrm{T}}\left(t-h_{1}(t)\right)\left(Q_{1}-Q_{2}\right) \times \\
& x\left(t-h_{1}(t)\right)-\left(1-d_{1}-d_{2}\right) x^{\mathrm{T}}(t-h(t)) Q_{2} x(t-h(t))
\end{aligned}
$$

where $Q_{1}-Q_{2} \geqslant 0$. Now, let

$$
\chi(t)=\left[\begin{array}{llll}
x^{\mathrm{T}}(t) & x^{\mathrm{T}}\left(t-h_{1}(t)\right) & x^{\mathrm{T}}(t-h(t)) & (E \dot{x}(t))^{\mathrm{T}}
\end{array}\right]^{\mathrm{T}} .
$$

Taking account of (15), (16) and (17), we have

$$
\dot{V}\left(x_{t}\right) \leqslant \chi^{\mathrm{T}}(t) \Phi \chi(t)
$$

where

$$
\Phi=\left[\begin{array}{cccc}
\Phi_{11} & \Phi_{12} & 0 & P \\
* & \Phi_{22} & \Phi_{23} & 0 \\
* & * & \Phi_{33} & 0 \\
* & * & * & \Phi_{44}
\end{array}\right]
$$

and

$$
\begin{aligned}
& \Phi_{11}=Q_{1}-\frac{1}{\bar{h}_{1}} E^{\mathrm{T}} Z_{1} E \\
& \Phi_{12}=\frac{1}{\bar{h}_{1}} E^{\mathrm{T}} Z_{1} E \\
& \Phi_{22}=-\frac{1}{\bar{h}_{1}} E^{\mathrm{T}} Z_{1} E-\frac{1}{\bar{h}_{2}} E^{\mathrm{T}} Z_{2} E-\left(1-d_{1}\right)\left(Q_{1}-Q_{2}\right) \\
& \Phi_{23}=\frac{1}{\bar{h}_{2}} E^{\mathrm{T}} Z_{2} E \\
& \Phi_{33}=-\frac{1}{\bar{h}_{2}} E^{\mathrm{T}} Z_{2} E-(1-d) Q_{2} \\
& \Phi_{44}=\bar{h}_{1} Z_{1}+\bar{h}_{2} Z_{2} .
\end{aligned}
$$

Now, let

$$
\begin{aligned}
& B=\left[\begin{array}{llll}
A_{0} & 0 & A_{d} & -I
\end{array}\right] \\
& F=\left[\begin{array}{l}
F_{0} \\
0 \\
F_{1} \\
F_{2}
\end{array}\right] .
\end{aligned}
$$

Then we can verify that $B \chi=0$. The matrix $M$ in (8) can be written as

$$
M=\Phi+F B+B^{\mathrm{T}} F^{\mathrm{T}}<0 .
$$

Applying Lemma 2, we have $\chi^{\mathrm{T}} \Phi \chi<0$ which implies that $\dot{V}\left(x_{t}\right)<0$. Thus, system (5) is asymptotically stable. 
Remark 1. When the time-delays $h_{1}(t)$ and $h_{2}(t)$ are constant, system (5) is regular, impulse free, and asymptotically stable, if only the conditions (6) and (8) hold with $d_{1}=d_{2}=0$.

Remark 2. In the derivation of our stability conditions, it can be clearly observed that neither model transformation is involved, nor bounding techniques for cross terms are required. Our paper presents a new approach based on Finsler's lemma to establish delay-dependent stability of singular systems with additive time-varying delays.

Remark 3. The condition developed in this theorem are delay-dependent. The result of this theorem can be easily extended to handle the case of systems with multiple additive time-varying delays described by

$$
E \dot{x}(t)=A_{0} x(t)+A_{d} x\left(t-\sum_{j=1}^{q} h_{j}(t)\right)
$$

where $0 \leqslant h_{j}(t) \leqslant \bar{h}_{j}, \dot{h}_{j}(t) \leqslant d_{j}, j=1, \cdots, q, \bar{h}=$ $\sum_{j=1}^{q} \bar{h}_{j}$, and $d=\sum_{j=1}^{q} d_{j}$.

And this theorem can be further extended to multiple delay case described by

$$
E \dot{x}(t)=A_{0} x(t)+\sum_{i=1}^{p} A_{d i} x\left(t-\sum_{j=1}^{q} h_{i j}(t)\right)
$$

where $0 \leqslant h_{i j}(t) \leqslant \bar{h}_{i j}, \dot{h}_{i j}(t) \leqslant d_{i j}, i=1, \cdots, p, j=$ $1, \cdots, q, \bar{h}_{i}=\sum_{j=1}^{q} \bar{h}_{i j}$, and $d_{i}=\sum_{j=1}^{q} d_{i j}$.

\section{Robust stability}

In this section, we will extend the results of the above section to uncertain systems (1).

Theorem 2. Given $\bar{h}_{1}>0$ and $\bar{h}_{2}>0$, the uncertain system (1) satisfying conditions (1) is regular, impulse free, and robustly stable, if there exist $n \times n$ matrices $P, F_{0}, F_{1}, F_{2}$, and $n \times n$ positive definite matrices $Q_{1}, Q_{2}, Z_{1}, Z_{2}$ and $Y \in \mathbf{R}^{p \times p}$, such that condition (6) and (7) hold and

$$
\left[\begin{array}{ccccc}
M_{11}+f_{11} & M_{12} & M_{13}+f_{12} & M_{14} & F_{0} D \\
* & M_{22} & M_{23} & M_{24} & 0 \\
* & * & M_{33}+f_{22} & M_{34} & F_{1} D \\
* & * & * & M_{44} & F_{2} D \\
* & * & * & * & -Y
\end{array}\right]<0 \quad(19)
$$

where $f_{11}=N_{0}^{\mathrm{T}} Y N_{0}, f_{12}=N_{0}^{\mathrm{T}} Y N_{d}, f_{22}=N_{d}^{\mathrm{T}} Y N_{d}, M_{i j}$ are given by (9).

Proof. Replacing $A_{0}$ and $A_{d}$ respectively by $A_{0}+$ $D F(t) N_{0}$ and $A_{d}+D F(t) N_{d}$ in condition (8) gives

$$
\left[\begin{array}{llll}
\tilde{M}_{11} & M_{12} & \tilde{M}_{13} & \tilde{M}_{14} \\
* & M_{22} & M_{23} & M_{24} \\
* & * & \tilde{M}_{33} & \tilde{M}_{34} \\
* & * & * & M_{44}
\end{array}\right]<0
$$

where

$$
\begin{aligned}
& \tilde{M}_{11}=Q_{1}-\frac{1}{\bar{h}_{1}} E^{\mathrm{T}} Z_{1} E+F_{0}\left(A_{0}+\right. \\
& \left.D F(t) N_{0}\right)+\left(A_{0}+D F(t) N_{0}\right)^{\mathrm{T}} F_{0}^{\mathrm{T}} \\
& \tilde{M}_{13}=F_{0}\left(A_{d}+D F(t) N_{d}\right)+\left(A_{0}+D F(t) N_{0}\right)^{\mathrm{T}} F_{1}^{\mathrm{T}}
\end{aligned}
$$

$$
\begin{aligned}
\tilde{M}_{14}= & P-F_{0}+\left(A_{0}+D F(t) N_{0}\right)^{\mathrm{T}} F_{2}^{\mathrm{T}} \\
\tilde{M}_{33}= & -\frac{1}{\bar{h}_{2}} E^{\mathrm{T}} Z_{2} E-\left(1-d_{1}-d_{2}\right) Q_{2}+ \\
& F_{1}\left(A_{d}+D F(t) N_{d}\right)+\left(A_{d}+D F(t) N_{d}\right)^{\mathrm{T}} F_{1}^{\mathrm{T}} \\
\tilde{M}_{34}= & -F_{1}+\left(A_{d}+D F(t) N_{d}\right)^{\mathrm{T}} F_{2}^{\mathrm{T}}
\end{aligned}
$$

which can be written as

$$
M+\operatorname{sym}\left\{\left[\begin{array}{c}
F_{0} \\
0 \\
F_{1} \\
F_{2}
\end{array}\right] D F(t)\left[\begin{array}{llll}
N_{0} & 0 & N_{d} & 0
\end{array}\right]\right\}<0
$$

where $\operatorname{sym}\{A\}=A+A^{\mathrm{T}}$. Applying Lemma 1, there exists $Y>0$ such as

$$
M+\left[\begin{array}{c}
F_{0} \\
0 \\
F_{1} \\
F_{2}
\end{array}\right] D Y^{-1} D^{\mathrm{T}}\left[\begin{array}{c}
F_{0} \\
0 \\
F_{1} \\
F_{2}
\end{array}\right]^{\mathrm{T}}+\left[\begin{array}{c}
N_{0}^{\mathrm{T}} \\
0 \\
N_{d}^{\mathrm{T}} \\
0
\end{array}\right] Y\left[\begin{array}{c}
N_{0}^{\mathrm{T}} \\
0 \\
N_{d}^{\mathrm{T}} \\
0
\end{array}\right]^{\mathrm{T}}<0
$$

The use of the Schur complement leads to the desired result.

Remark 4. In the case of constant delays $h_{1}(t)$ and $h_{2}(t)$, we require only the conditions (6) and (19) with $d_{1}=$ $d_{2}=0$ for checking regularity, impulse free state, and robust stability for the system (1).

\section{$5 \quad$ Numerical examples}

In this section, we aim to demonstrate the effectiveness and the less conservatism of the proposed approach.

Example $1^{[21]}$. Consider the following singular system with constant time delay,

$$
\begin{gathered}
E=\left[\begin{array}{ll}
1 & 0 \\
0 & 0
\end{array}\right] \\
A_{0}=\left[\begin{array}{cc}
0.6341 & 0.5413 \\
-0.6121 & -1.1210
\end{array}\right] \\
A_{d}=\left[\begin{array}{cc}
-0.4500 & 0 \\
0 & -0.1210
\end{array}\right]
\end{gathered}
$$

Letting $d_{1}=d_{2}=0$ and applying Theorem 1, our purpose is to find the upper bound $\bar{h}_{1}$ of delay $h_{1}(t)$ when $\bar{h}_{2}$ is given, or to compute the upper bound $\bar{h}_{2}$ of delay $h_{2}(t)$ when $\bar{h}_{1}$ is given. The upper bound $\bar{h}$ is obtained by summing the two delay bounds $\bar{h}_{1}$ and $\bar{h}_{2}$. It has been proved in Theorem 1 of [30] that its result is equivalent to those of [31-34]. For comparison, we report the results in Table 1.

It is well known that the results of time-varying delays are more conservative than that of time invariant ones. In the existing literature results ${ }^{[8,10,13,19,21,30,32-37]}$, the time delay considered is constant, and the delay is timevarying in [31]. However, our result is derived for timevarying delay and gives less conservative bounds.

From Table 1, it can be seen that our method gives less conservative results. 
Table 1 Comparison of delay-dependent stability conditions of Example 1

\begin{tabular}{|c|c|c|c|c|c|c|c|}
\hline Method & \multicolumn{7}{|c|}{ Upper bound $\bar{h}=\bar{h}_{1}+\bar{h}_{2}$} \\
\hline Reference [35] & \multicolumn{7}{|c|}{-} \\
\hline Reference $[8]$ & \multicolumn{7}{|c|}{2.1328} \\
\hline Reference $[10,13]$ & \multicolumn{7}{|c|}{2.1372} \\
\hline Reference $[36,37]$ & \multicolumn{7}{|c|}{2.4841} \\
\hline Reference $[21]$ & \multicolumn{7}{|c|}{2.4865} \\
\hline Reference $[30,31-34]$ & \multicolumn{7}{|c|}{2.4865} \\
\hline Reference [19] & \multicolumn{7}{|c|}{2.4865} \\
\hline & \multicolumn{4}{|c|}{ Upper bound $\bar{h}_{2}$ for given $\bar{h}_{1}$} & \multicolumn{3}{|c|}{ Upper bound $\bar{h}_{1}$ for given $\bar{h}_{2}$} \\
\hline \multirow[t]{2}{*}{ Theorem 1} & $\bar{h}_{1}=0.00001$ & $\bar{h}_{1}=0.4$ & $\bar{h}_{1}=1$ & $\bar{h}_{1}=1.5$ & $\bar{h}_{2}=1$ & $\bar{h}_{2}=1.4$ & $\bar{h}_{2}=1.8$ \\
\hline & 2.4865 & 2.1008 & 1.5153 & 1.0167 & 1.5165 & 1.1167 & 0.7096 \\
\hline
\end{tabular}

Table 2 Comparison of delay-dependent robust stability conditions of Example 2

\begin{tabular}{ccccccc}
\hline$\alpha$ & 0.25 & 0.30 & 0.35 & 0.40 & 0.45 \\
\hline Reference [36] & 0.4209 & 0.3939 & 0.3637 & 0.3279 & 0.2817 & 0.7262 \\
Reference [14] & 0.8087 & 0.7942 & 0.7689 & 0.7438 & 0.6521 \\
Reference [16] & 0.8514 & 0.8249 & 0.7924 & 0.6641 & 0.8448 & 0.5110 \\
Reference [33] & 0.8962 & 0.8787 & 0.8616 & 0.8448 & 0.8283 \\
Theorem 2 for $\bar{h}_{1}=0.00001$ & 0.8962 & 0.8787 & 0.8616 & 0.8737 & 0.8555 \\
Theorem 2 for $\bar{h}_{1}=0.4$ & 0.9298 & 0.9109 & 0.8922 & 0.8374 \\
\hline
\end{tabular}

Example $2^{[16]}$. Consider the uncertain singular delay system (1) with

$$
\begin{gathered}
E=\left[\begin{array}{ll}
2 & 0 \\
0 & 0
\end{array}\right] \\
A_{0}=\left[\begin{array}{cc}
1 & 0 \\
0 & -2
\end{array}\right] \\
A_{d}=\left[\begin{array}{cc}
-2.4 & 2 \\
0 & 1
\end{array}\right]
\end{gathered}
$$

where $\left\|\Delta A_{0}\right\| \leqslant \alpha,\left\|\Delta A_{d}\right\| \leqslant \alpha(\alpha>0)$, and the delay is constant. The uncertainty is written as $D=\alpha I$, $N_{0}=N_{d}=0.5 I$. In order to apply our results, we set $d_{1}=d_{2}=0$, we compute $\bar{h}_{2}$ when $\bar{h}_{1}=0.4$ and $\bar{h}_{1}=0.00001$, and we deduce the delay bound $\bar{h}$. Table 2 gives the comparison of the maximum allowed delay $\bar{h}$ for various values of the parameter $\alpha$. It is clear that the conditions in this paper give better results than those in $[14,16,33,36]$, showing the advantage of the stability result in this paper.

\section{Conclusions}

Delay-dependent conditions are presented in terms of linear matrix inequalities (LMIs) for asymptotic stability and robust stability of singular systems with two additive timevarying delays. The LMIs proposed have been obtained by utilizing a Lyapunov Krasovskii functional. Numerical examples are given to illustrate the effectiveness of the proposed method and to show that our criteria give less conservative results.

\section{References}

[1] S. M. Saadni, M. Chaaban, D. Mehdi. Robust stability and stabilization of a class of singular systems with multiple time-varying delays. Asian Journal of Control, vol. 8, no. 1, pp. 1-11, 2006.
[2] L. Dai. Singular Control Systems, New York, USA: Springer-Verlag, 1989.

[3] F. L. Lewis. A survey of linear singular systems. Circuits, Systems, and Signal Processing, vol. 5, no. 1, pp. 3-36, 1986.

[4] S. Ma, C. Zhang, S. Zhu. Robust stability for discrete-time uncertain singular Markov jump systems with actuator saturation. IET Control Theory and Applications, vol. 5, no. 2, pp. 255-262, 2011.

[5] S. Ma, E. K. Boukas. Stability and robust stabilisation for uncertain discrete stochastic hybrid singular systems with time delay. IET Control Theory and Applications, vol.3, no. 9, pp. 1217-1225, 2009.

[6] S. P. Ma, E. K. Boukas, Y. Chinniah. Stability and stabilization of discrete-time singular Markov jump systems with time-varying delay. International Journal of Robust and Nonlinear Control, vol. 20, no. 5, pp. 531-543, 2010.

[7] S. Y. Xu, G. Feng. New results on $H_{\infty}$ control of discrete singularly perturbed systems. Automatica, vol. 45, no. 10, pp. 2339-2343, 2009.

[8] E. Fridman. Stability of linear descriptor systems with delay: A Lyapunov-based approach. Journal of Mathematical Analysis and Applications, vol. 273, no. 1, pp. 24-44, 2002.

[9] E. Fridman. A Lyapunov-based approach to stability of descriptor systems with delay. In Proceedings of the 40th IEEE Conference on Decision and Control, IEEE, Orlando, USA, vol. 3, pp. 2850-2855, 2001.

[10] E. Fridman, U. Shaked. $H_{\infty}$ control of linear state-delay descriptor systems: An LMI approach. Linear Algebra and Its Applications, vol. 351-352, pp. 271-302, 2002.

[11] E. K. Boukas, Z. K. Liu. Delay-dependent stability analysis of singular linear continuous-time system. IEE Proceedings: Control Theory \& Applications, vol. 150, no. 4, pp. 325-330, 2003

[12] E. K. Boukas, N F Almuthairi. Delay-dependent stabilization of singular linear systems with delays. International Journal of Innovative Computing, Information and Control, vol. 2, no. 2, pp. 283-291, 2006.

[13] F. Yang, Q. L. Zhang. Delay-dependent $H$-infinity control for linear descriptor systems with delay in state. Journal of Control Theory and Applications, vol.3, no. 1, pp. 76-84, 2005. 
[14] H. L. Gao, S. Q. Zhu, Z. L. Cheng, B. G. Xu. Delaydependent state feedback guaranteed cost control for uncertain singular time-delay systems. In Proceedings of the 44th IEEE Conference on Decision and Control, and 2005 European Control Conference, IEEE, Seville, Spain, pp. 43544359, 2005.

[15] S. Y. Xu, P. Van Dooren, R. Stefan, J. Lam. Robust stability and stabilization for singular systems with state delay and parameter uncertainty. IEEE Transactions on Automatic Control, vol. 47, no. 7, pp. 1122-1128, 2002.

[16] Z. G. Wu, W. N. Zhou. Delay-dependent robust stabilization for uncertain singular systems with state delay. Acta Automatica Sinica, vol. 33, no. 7, pp.714-718, 2007. (in Chinese)

[17] X. Z. Dong, Q. L. Zhang. Robust input-output energy decoupling for uncertain singular systems. International Journal of Automation and Computing, vol. 2, no. 1, pp. 37-42, 2005.

[18] C. Y. Yang, X. Jing, Q. L. Zhang, L. N. Zhou. Practical stability analysis and synthesis of linear descriptor systems with disturbances. International Journal of Automation and Computing, vol. 5, no. 2, pp. 138-144, 2008.

[19] X. Sun, Q. L. Zhang, C. Y. Yang, Z. Su, Y. Y. Shao. An improved approach to delay-dependent robust stabilization for uncertain singular time-delay systems. International Journal of Automation and Computing, vol.7, no. 2, pp. 205$212,2010$.

[20] J. E. Feng, S. Q. Zhu, Z. L. Cheng. Guaranteed cost control of linear uncertain singular time-delay systems. In Proceedings of the 41st IEEE Conference on Decision and Control, IEEE, Las Vegas, USA, vol. 2, pp. 1802-1807, 2002.

[21] Z. G. Wu, H. Y. Su, J. Chu. Improved results on delaydependent $H_{\infty}$ control for singular time-delay systems. Acta Automatica Sinica, vol. 35, no. 8, pp. 1101-1106, 2009. (in Chinese)

[22] M. Fang. Delay-dependent robust $H_{\infty}$ control for uncertain singular systems with state delay. Acta Automatica Sinica, vol. 35 , no. 1 , pp. 65-70, 2009. (in Chinese)

[23] H. J. Gao, T. W. Chen, J. Lam. A new delay system approach to network based control. Automatica, vol. 44, no. 1, pp. 39-52, 2008.

[24] J. Lam, H. J. Gao, C. H. Wang. Stability analysis for continuous systems with two additive time-varying delay components. Systems \& Control Letters, vol. 56, no. 1, pp. 16-24, 2007.

[25] R. Dey, G. Ray, S. Ghosh, A. Rakshit. Stability analysis for continuous system with additive time-varying delays: A less conservative result. Applied Mathematics and Computation, vol. 215, no. 10, pp. 3740-3745, 2010.

[26] H. X. Wu, X. F. Liao, W. Feng, S. T. Guo, W. Zhang. Robust stability analysis of uncertain systems with two additive time-varying delay components. Applied Mathematical Modelling, vol. 33, no. 12, pp. 4345-4353, 2009.

[27] I. R. Peterson, C. V. Hollot. A Riccati equation approach to the stabilization of uncertain linear systems. Automatica, vol. 22, no. 4, pp. 397-411, 1986.

[28] M. C. de Oliveira, R. E. Skelto. Stability tests for constrained linear systems. Perspectives in Robust Control of Lecture Notes in Control and Information Science, S. O. Reza Moheimani, Springer-Verlag, New York, USA, vol. 268, pp. 241-257, 2001.

[29] T. Li, B. Yang, J. C. Wang, C. Q. Zhong. On stability for neutral differential systems with mixed time-varying delay arguments. In Proceedings of the 42nd IEEE Conference on Decision and Control, IEEE, Maui, HI, USA, vol. 5, pp. 5066-5067, 2003.
[30] Y. F. Feng, X. L. Zhu, Q. L. Zhang. Delay-dependent stability criteria for singular time-delay systems. Acta Automatica Sinica, vol. 36, no. 3, pp. 433-437, 2010. (in Chinese)

[31] D. Yue, Q. L. Hang. A delay-dependent stability criterion of neutral systems and its application to a partial element equivalent circuit model. IEEE Transactions on Circuits and Systems II: Express Briefs, vol. 51, no. 12, pp. 685-689, 2004

[32] S. Y. Xu, J. Lam, Y. Zou. An improved characterization of bounded realness for singular delay systems and its applications. International Journal of Robust and Nonlinear Control, vol. 18, no. 3, pp. 263-277, 2008.

[33] S. Zhu, C. Zhang, Z. Cheng, J. Feng. Delay-dependent robust stability criteria for two classes of uncertain singular time-delay systems. IEEE Transactions on Automatic Control, vol. 52, no. 5, pp. 880-885, 2007.

[34] Z. Wu, W. N. Zhou. Delay-dependent robust $H_{\infty}$ control for uncertain singular time-delay systems. IET Control Theory and Applications, vol. 1, no. 5, pp. 1234-1241, 2007.

[35] E. K. Boukas. Singular linear systems with delay: $H_{\infty}$ stabilization. Optimal Control Applications and Methods, vol. 28, no. 4, pp. 259-274, 2007.

[36] R. X. Zhong, Z. Yang. Delay-dependent robust control of descriptor systems with time delay. Asian Journal of Control, vol. 8, no. 1, pp. 36-44, 2006.

[37] S. Q. Zhu, Z. L. Cheng, J. N. Feng. Delay-dependent robust stability criterion and robust stabilization for uncertain singular time-delay systems. In Proceedings of American Control Conference, IEEE, Portland, USA, pp. 28392844, 2005.

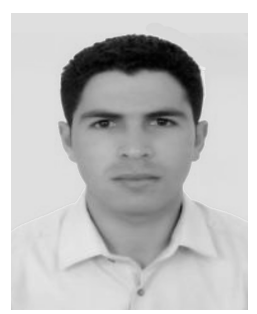

N. Chaibi received the M. Eng. degree in automated industrial systems from Faculty of Sciences, University of Sidi Mohammed Ben Abdellah, Morocco in 2009.

His research interests include stability and stabilization of singular systems with time delays.

E-mail: ieea_nour@yahoo.fr (Corresponding author)

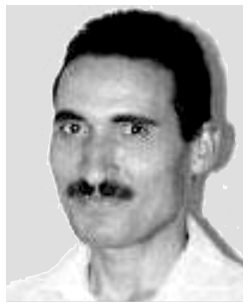

E. H. Tissir received the diploma of higher studies and doctorate of state degree from Faculty of Sciences, University Sidi Mohammed Ben Abellah, Morocco in 1992 and 1997, respectively. He is now a professor at the University Sidi Mohammed Ben Abellah.

His research interests include robust and $H_{\infty}$ control, singular systems, switched and time delay systems, and systems with sat-

urating actuators.

E-mail: elh_tissir@yahoo.fr

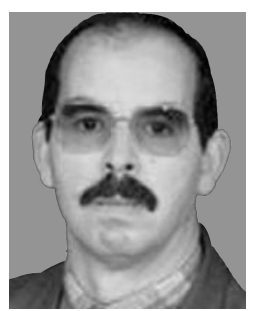

A. Hmamed received his Doctorate of state degree in electrical engineering from the Faculty of Sciences, Rabat, Morocco in 1985. Since 1986, he has been with the Department of Physics, Faculty of Sciences, University Sidi Mohammed Ben Abellah, where he is currently a full professor.

His research interests include delay systems, stability theory, systems with constraints, and 2D systems and applications. E-mail: hmamed_abdelaziz@yahoo.fr 